\title{
The Vessel Schedule Recovery Problem (VSRP) - A MIP model for handling disruptions
} in liner shipping

Pisinger, David; Brouer, Berit Dangaard; Dirksen, Jakob; Plum, Christian Edinger Munk; Vaaben, Bo Valdemar

\section{Published in:}

Proceedings of the 28th European Conference on Operational Research

Publication date:

2016

Document Version

Publisher's PDF, also known as Version of record

Link back to DTU Orbit

Citation (APA):

Pisinger, D., Brouer, B. D., Dirksen, J., Plum, C. E. M., \& Vaaben, B. V. (2016). The Vessel Schedule Recovery Problem (VSRP) - A MIP model for handling disruptions in liner shipping. In Proceedings of the 28th European Conference on Operational Research (pp. 30-30)

\section{General rights}

Copyright and moral rights for the publications made accessible in the public portal are retained by the authors and/or other copyright owners and it is a condition of accessing publications that users recognise and abide by the legal requirements associated with these rights.

- Users may download and print one copy of any publication from the public portal for the purpose of private study or research.

- You may not further distribute the material or use it for any profit-making activity or commercial gain

- You may freely distribute the URL identifying the publication in the public portal 
the hierarchy. On the other hand, SMAA permits to compute the probability that an alternative gets a particular position in the recommended ranking, or probability that an alternative is preferred to another one, in all nodes of the hierarchy of criteria. We apply the proposed methodology to the ranking of universities.

\section{2 - The ranking of Polish research institutions based on the distance measure function in the value space Piotr Zielniewicz}

The paper presents a method combining the robust ordinal regression approach and the idea of ranking alternatives based on the distance measure function. In this method, the preference model is composed of a set of additive value functions compatible with the preference information provided by the decision maker in the form of pairwise comparisons of reference alternatives. From among many forms of an additive preference model, we consider the model having as simple form as possible, i.e., the model that is the "closest to linear". We define a function representing closeness to the reference point (the ideal solution), however not in the criteria space, but in the value space. A set of mix-integer linear programming problems is solved to determine the minimum distance scores of each alternative (in this case research institution) on the set of compatible value functions. Finally, the obtained distance scores are used to rank all research institutions.

\section{3 - Multi-criteria repair/recovery solutions for territory as-} signment problem

Oumaima Khaled, Michel Minoux, Vincent Mousseau, Xavier Ceugniet

In this talk we investigate solution recovery/repair issues in the context of a territory assignment problem where a number of salespersons (SPs) have to be assigned to customers in a given geographical area. Each salesperson operates from a given base office. The customers are grouped into clusters, the characteristics of which are supposed to be known (location, type(s) of product(s), etc.) and a SP has to be assigned to each customer cluster(CC). The main objective of an assignment is the minimization of the sum (over all CC) of the distance between the center of the CC and the base office of the SP assigned to it. The recovery/repair scenarios addressed assume that a first (optimal) assignment has already been determined, but because of the dynamic structure of the market context, various unexpected events can occur requiring reconsideration of this initial assignment. The question of "best repair", i.e., determining a new solution to the perturbed problem in order to come up with both a reduced increase in the total distance criterion and limited changes to be carried out in the assignment, typically involves multiple criteria such as the number of CCs impacted by the change, the number of SPs impacted, etc. We show that this repair problem can be formalized as a multi-objective integer linear programming problem minimizing a specified function of the various repair criteria. Numerical results illustrating the relevance of the proposed model will be given and discussed.

4 - Dominance based Monte-Carlo algorithm for multicriteria ordinal classification

Tom Denat, Meltem Ozturk

In this document we are studying a multi-criteria method for preference elicitation and ordinal classification. In literature there are several methods adapted to ordinal classification, such as ELECTRE TRI, rule based or additive-utility methods. Such methods use decision parameters (thresholds, weights ...) which must be elicitated (directly or indirectly) from the decision maker. The specificity of our approach is to be probabilistic, based on a Monte-Carlo principle and thus not requiring sophisticated decision parameters. Hence, we do not assume that the decision maker's reasoning follows some well-known and explicitly described rules or logic system. The only two assumptions that we made are that monotonicity should be respected as well as the classification examples given by the decision maker (learning set). Three variants of our methods will be presented. We proved that for all the variants the learning set is respected and the result converges a.s. and that for two variants monotonicity is respected. We saw practically that the convergence of the result is quite effective. We then compared our algorithm to three other MCDA elicitation methods (UTADIS, MRSort, DRSA) through a k-fold validation. This test was based on two sets of real data: judgment on the severity of potential accidental pollution (data from ecology specialists) and an ethic judgment on companies (from individuals). Our algorithm gets on these data sensibly better results than the others.
MB-09

Monday, 10:30-12:00 - Building CW, 1st floor, Room 12

\section{OR Applications in Industry}

\section{Stream: OR Applications in Industry Chair: Geir Hasle}

\section{1 - Flexibility value in Transmission Expansion Planning in} Colombia using Real Options.

\section{Alvin Henao Perez, Enzo Sauma, Angel Gonzalez}

This paper presents an estimation of the the value of introducing flexibility in the Colombian Transmission Expansion Planning (TEP). This approach uses Real Options (RO) applied on a stylized version of Colombian electricity network. The transmission expansion process is split in a fixed expansion and a flexible expansion. The latter is used as an adapting mechanism to handle demand growth rates higher than expected. Results show the value that flexibility in Colombian TEP process has.

\section{2 - Optimization for a Semi-Automated Warehouse}

\section{Ali Can Özcan, Bahar Yetis Kara, Ozlem Cavus, Tolga}

Dizdarer, Onur Altıntaş, Hakan Gultekin

This study focuses on the optimization of a Sorter system in a facility of Ekol Logistics. The system is composed of conveyors and automated baskets that handle product sorting, and manual labor that handles the product movement in between. Products are fed to the Sorter through conveyors and then manually transferred to automatic baskets that will drop them to their correct packages. The aim of this study is to create optimal feeding schedules to design a balanced flow of products to the system and to generate a continuous feeding capability that will eliminate the unnecessary idle time in between the waves of operation. Two models are proposed for optimal feeding schedule: a mixed integer optimization model and a simulation model. In addition to these models, a heuristic solution is also suggested. The models and the heuristic algorithm use company's operation data and product demands to find the hourly feeding schedule. They schedule workers for 3 shifts and assign the labor to required locations. The suggested models can be modified to be used in different environments with different sets of constraint.

\section{3 - Applied OR at SINTEF}

Geir Hasle

SINTEF is a Norwegian cross-disciplinary contract research institute that aims at bridging the gap between academia and industry. OR activities are found in many organizational units. In this talk, I will present examples of OR activities in the Group of Optimization, Department of Applied Mathematics. Examples include logistics simulation and optimization in railway and bus transportation, air traffic management, vehicle routing, and healthcare.

\section{MB-10}

Monday, 10:30-12:00 - Building CW, ground floor, Room 1

\section{Meet the Editors of EJOR}

Stream: EURO Awards and Journals

Chair: Roman Slowinski

Chair: Immanuel Bomze

Chair: Robert Dyson

Chair: José Fernando Oliveira

Chair: Ruud Teunter

Chair: Emanuele Borgonovo

\section{1 - Some facts about the European Journal of Operational} Research (EJOR)

Roman Slowinski, Immanuel Bomze, Emanuele Borgonovo, Robert Dyson, José Fernando Oliveira, Ruud Teunter

The editors of EJOR will give some characteristics of the journal, and will explain their approach to evaluation and selection of articles. They will point out topics of OR which recently raised the highest interest. Two other presentations in the session will be done by authors of representative and highly cited papers published recently in EJOR in two categories: theory \& methodology, and innovative application of OR. In the last part of the session, the editors will answer some general questions from the audience. 
2 - Competitive Food Supply Chain Networks with Application to Fresh Produce

Anna Nagurney, Min $\mathrm{Yu}$

In this paper, we develop a network-based food supply chain model under oligopolistic competition and perishability, with a focus on fresh produce. The model incorporates food deterioration through the introduction of arc multipliers, with the inclusion of the discarding costs associated with the disposal of the spoiled food products. We allow for product differentiation due to product freshness and food safety concerns, as well as the evaluation of alternative technologies associated with various supply chain activities. We then propose an algorithm with elegant features for computation. A case study focused on the cantaloupe market is investigated within this modeling and computational framework, in which we analyze different scenarios prior/during/after a foodborne disease outbreak.

We relate the model to several other supply chain network models of perishable products, including medical nuclear ones, and demonstrate how the original model can also be adapted to capture quality issues over time in food supply chains.

3 - The Vessel Schedule Recovery Problem (VSRP) - A MIP model for handling disruptions in liner shipping

David Pisinger, Berit Dangaard Brouer, Jakob Dirksen, Christian Edinger Munk Plum, Bo Vaaben

Containerized transport by liner shipping companies is a multi billion dollar industry carrying a major part of the world trade between suppliers and customers. The liner shipping industry has come under stress in the last few years due to the economic crisis, increasing fuel costs, and capacity outgrowing demand. The push to reduce $\mathrm{CO} 2$ emissions and costs have increasingly committed liner shipping to slow-steaming policies. This increased focus on fuel consumption, has illuminated the huge impacts of operational disruptions in liner shipping on both costs and delayed cargo. Disruptions can occur due to adverse weather conditions, port contingencies, and many other issues. A common scenario for recovering a schedule is to either increase the speed at the cost of a significant increase in the fuel consumption or delaying cargo. Advanced recovery options might exist by swapping two port calls or even omitting one. We present the Vessel Schedule Recovery Problem (VSRP) to evaluate a given disruption scenario and to select a recovery action balancing the trade off between increased bunker consumption and the impact on cargo in the remaining network and the customer service level. It is proven that the VSRP is NPNP-hard. The model is applied to four real life cases from Maersk Line and results are achieved in less than 5 seconds with solutions comparable or superior to those chosen by operations managers in real life. Cost savings of up to $58 \%$ may be achieved by the suggested solutions compared to realized recoveries of the real life cases.

\section{MB-11}

Monday, 10:30-12:00 - Building CW, 1st floor, Room 127

\section{Neural Networks and Applications}

\author{
Stream: Fuzzy Optimization - Systems, Networks and Ap- \\ plications \\ Chair: Kiran Anwar \\ Chair: Jun-Der Leu
}

\section{1 - An Empirical Comparison of Different RBF Neural Net- work Training Algorithms for Classification}

Tiny Du Toit

Radial Basis Function Neural Networks (RBFNNs) use Radial Basis Functions as their activation functions. The final network output is a linear combination of the Radial Basis Functions of the neuron parameters and the inputs. RBFNNs are popular for time series prediction, curve fitting, control, function approximation, signal processing, and classification. A RBFNN has several distinctive features making it different from other types of neural networks. These features include a more compact architecture, faster learning, and universal approximation. Several RBFNN training algorithms have been proposed in the literature. Amongst them are the Genetic algorithm, Kalman filtering algorithm, the gradient descent algorithm, and the Artificial Bee Colony algorithm. In this research, a hybrid conjugate gradient descent method is compared to the above-mentioned algorithms. Well known classification problems from the UCI Machine Learning Repository are used in the experiments. Results indicate that the hybrid conjugate gradient descent method outperforms the other algorithms on simple RBFNN models based on the percentage of correctly classified samples (PCCS) metric as well as the standard deviations of the PCCS.

2 - Plantar Pressure Identification and Differentiation Using an Artificial Neuromolecular System

JongChen Chen

Inappropriate use of foot may result in diseases on foot. The aim of this study is to investigate the relation between plantar pressures and center of gravity (COG), from which we can use to differentiate various features of users, to analyze plantar pressure of users with different motions, and finally to investigate users' plantar pressure under different situations. Our ultimal goal is to develop a customerized plantar pressure system for different people, time, and needs. To collect the data of human plantar pressure data, for each foot, five piezoresistive force sensors were embedded into an insole ( 10 sensors for both feet). These sensors were linked with an Arduino, a family of single-board microcontrollers, was used to input, process, and output data between the piezoresistive force sensors and the computer. The floor-based device Model BP 5050 was used to measure gravity of center (GOC) while wearing the in-shoe sensor (device) fitted in a shoe on the force plate for collecting the data of GOC and plantar pressure. An artificial neuromolecular system earlier constructed in our lab was used to differentiate behavior modes of different users. A conclusive result was that the biometric features possessed by each user were quite different that allowed us to separate one from another. Based on the plantar pressure data of each individual, we were able to differentiate normal/abnormal behaviors.

3 - Predicting the Direction of a Stock Index Using Artificial Neural Networks and Evidential Reasoning Dong-Ling $\mathrm{Xu}$, Chen You

This paper focuses on the prediction of the direction, either up or down, of Shanghai Stock Exchange Composite Index using Artificial Neural Networks (ANNs) and Evidential Reasoning (ER) models. Factors affecting the movement of a stock index are first identified as the inputs to the models. After model building and optimal tuning of model parameters, it is shown that ANN models perform better in predicting the "seen" sample data, while the ER model perform more accurately for forecasting new observations.

A new concept, strength of predictions, is introduced in the paper. It is defined as $\mid \mathrm{P}($ up $)$ - $\mathrm{P}($ down $) \mid$ or the absolute difference between the predicted probabilities of a stock's price going up and down. It is shown that for those stocks having high prediction strengths, the models have high prediction accuracy. Such a finding leads to the derivation of a selective trading strategy which generates high monetary gains.

As ER is a probabilistic reasoning process which extends Bayesian reasoning by considering the reliabilities and weights of those factors affecting a stock market movement, the prediction performance of ER and Bayesian models are also compared. ER shows a better performance. The paper concludes with a discussion of the advantages and disadvantages of ANN and ER models, and future research directions in the area.

4 - Design and Development of a Heuristic Based Evaluation function for General Game Playing

Kiran Anwar, Sobia Khalid, Sana Yousuf

General Game Playing (GGP) refers to formation of artificial intelligent agent that can play several games if game rules are provided. In this paper a heuristic based evaluation function called Goal Seeking Dead End Avoidance (GOSEDA) designed for GGP player is presented. The emphasis is to make player's performance better such that it does not only play games but also win games. The algorithm GOSEDA focuses on avoidance of dead-end during match play. GOSEDA algorithm explores the game tree and allows the player to select only those nodes during game play that have maximum number of moves. GOSEDA algorithm is evaluated using four benchmark Game Description Language (GDL) games. Experiments have been performed by connecting the player with Game Controller provided by Stanford logic group of Stanford University. In each game the player plays a match against Random Player of the Game Controller provided by the Stanford University logic group of research. The basic parameter for measuring performance of the algorithm designed for the GGP player is score of the player. The algorithm takes more time to play or win the game than its rival player strategy but still shows remarkable performance for two of the benchmark games. 